\title{
X-ray and UV Views of Hot Gas in Planetary Nebulae
}

\author{
You-Hua Chu, Martín A. Guerrero, and Robert A. Gruendl \\ Astronomy Department, University of Illinois, 1002 W. Green Street, \\ Urbana, IL 61801, USA
}

\begin{abstract}
The interior of a planetary nebula (PN) is expected to be filled with shocked fast wind from the central star. This hot gas plays the most important role in the dynamical evolution of the PN; however, its physical conditions are not well-known because useful X-ray and farUV observations were not available until the advent of Chandra, XMMNewton, and FUSE. This paper reviews X-ray observations of the hot gas in PN interiors and far-UV observations of the interfaces between the hot gas and the dense nebular shells.
\end{abstract}

\section{Introduction - Origin of Hot Gas in PNe}

It is commonly accepted that $\mathrm{PNe}$ are formed by the current fast stellar wind sweeping up the former slow asymptotic giant branch (AGB) wind. In this interacting-stellar-winds scenario (Kwok, Purton, \& FitzGerald 1978), the physical structure of a PN is similar to that of an interstellar wind-blown bubble as modeled by Weaver et al. (1977). The interior of a PN is filled with shocked fast wind reaching temperatures of $10^{7}-10^{8} \mathrm{~K}$, and this hot gas is confined within a $10^{4} \mathrm{~K}$ nebular shell consisting of swept-up AGB wind. The shocked fast wind per se has too low a density to emit appreciably in X-rays. At the interface between the hot interior and the cool nebular shell, heat conduction lowers the temperature and mass evaporation raises the density of the hot interior gas, producing optimal conditions for X-ray emission. Therefore, soft X-ray emission is expected from the $10^{6} \mathrm{~K}$ gas near the interface.

The physical processes taking place at the interface have been mostly a theoretical topic. The interface physics is expected to be complicated by the presence of magnetic field and turbulences. To thoroughly understand the interface physics, observations of the transition region are needed. While the $10^{6} \mathrm{~K}$ gas can be observed in X-rays, the adjacent layer of $10^{5} \mathrm{~K}$ gas can be observed only in the UV and far-UV through spectral lines of collisionally ionized C, N, and $\mathrm{O}$ at high ionization stages.

\section{X-ray Views of Hot Gas in Planetary Nebulae}

$\mathrm{X}$-ray observations of the hot gas in $\mathrm{PN}$ interiors can be confused by the X-ray emission from the central star of a PN (CSPN). A hot CSPN with a low opacity in its atmosphere can be a soft X-ray emitter, while a CSPN with a late-type 
companion may show a coronal X-ray source. The photospheric emission from a hot CSPN peaks below $0.1 \mathrm{keV}$ and can be spectroscopically discerned from thin plasma emission. On the other hand, the coronal emission from a late-type companion may show similar spectral shapes to plasma emission; thus, emission from the hot gas in $\mathrm{PN}$ interiors can be unambiguously confirmed only if it is spatially resolved. The quest for diffuse X-ray emission from hot gas in PN interiors is long and difficult, but significant progress has been made as the sensitivity and resolution of X-ray detectors have improved.

\subsection{X-ray Observing Facilities}

Since 1978, several X-ray satellites have been launched with imaging instruments sensitive to soft X-rays in the energy range $0.1-2 \mathrm{keV}$, where X-ray emission from $\mathrm{PNe}$ is expected. Table 1 summarizes the X-ray observing facilities available in the past 25 years. The earlier imaging detectors with spectral capabilities are mostly proportional counters with inherently low angular resolution ( $\geq 30^{\prime \prime}$ ) and spectral resolution (e.g., $E / \Delta E \sim 2.2$ at $1 \mathrm{keV}$ for $R O S A T$ PSPC). This situation is vastly improved by the advent of solid-state and CCD detectors, e.g., Chandra ACIS has an angular resolution of $1^{\prime \prime}$ and a spectral resolution of $E / \Delta E \sim 10$ at $1 \mathrm{keV}$. The currently available Chandra ACIS and XMM-Newton EPIC, with their unprecedented spatial and spectral resolutions and sensitivity, are ideal for X-ray observations of PNe.

Table 1. X-ray Observing Facilities Available in Recent Years

\begin{tabular}{ccccc}
\hline $\begin{array}{c}\text { Satellite } \\
\text { Name }\end{array}$ & $\begin{array}{c}\text { Period of } \\
\text { Operation }\end{array}$ & Instrument & $\begin{array}{c}\text { Angular } \\
\text { Resolution }\end{array}$ & $\begin{array}{c}\text { Spectral } \\
\text { Resolution }\end{array}$ \\
\hline Einstein & $1978-1981$ & IPC & $120^{\prime \prime}$ & Low \\
& & HRI & $5^{\prime \prime}$ & None \\
EXOSAT & $1983-1986$ & LE & $30^{\prime \prime}$ & None \\
ROSAT & $1990-1998$ & PSPC & $30^{\prime \prime}$ & Low \\
& & HRI & $5^{\prime \prime}$ & None \\
ASCA & $1993-2000$ & SIS & $150^{\prime \prime}$ & High \\
Chandra & $1999-$ pres. & ACIS & $1^{\prime \prime}$ & High \\
& & HRC & $0^{\prime \prime} 5$ & None \\
XMM-Newton & $1999-$ pres. & EPIC & $6^{\prime \prime}$ & High \\
\hline \hline
\end{tabular}

\subsection{X-ray Observations of $\mathrm{PNe}$}

The earliest X-ray observations of PNe were aimed to detect the photospheric emission from the CSPNs. While Einstein observed PNe several years before $E X O S A T$, the first report of X-ray emission from a CSPN was made with EXOSAT observations of NGC 1360 by de Korte et al. (1985). Using Einstein archival data of PNe, Tarafdar \& Apparao (1988) reported X-ray emission from NGC 246, NGC6853, NGC 7293, and A 33. Similarly, Apparao \& Tarafdar (1989) used the EXOSAT archive and reported X-ray emission from NGC 1535, NGC 3587, NGC 4361, and A 36. The reported emission from A 33 and NGC 1535 was later shown by ROSAT observation to arise from background X-ray sources (Conway \& Chu 1997; Chu, Gruendl, \& Conway 1998). 
The search for diffuse X-ray emission from hot gas in PN interiors was not attempted until ROSAT became available. During the first six months of the ROSAT mission, a PSPC detector was used to scan the whole sky. Using this ROSAT All-Sky Survey, Kreysing et al. (1992) reported diffuse X-ray emission from a number of PNe. Unfortunately, their work was plagued by low $\mathrm{S} / \mathrm{N}$ ratios in the data, electronic ghost image of the PSPC detectors at energies below $0.2 \mathrm{keV}$, and mis-identification of nearby or companion coronal sources. After careful scrutiny, the only object in their sample to show X-ray emission marginally more extended than the point spread function of the PSPC was NGC 6543.

During the ROSAT mission, pointed observations of a large number of PNe were made, but only the few positive detections were reported, for example, NGC 246, NGC 1360 (Hoare et al. 1995), NGC 3587 (Chu, Gruendl, \& Conway 1998), and NGC 7293 (Leahy, Zhang, \& Kwok 1994). When the ROSAT mission concluded in 1998, the ROSAT archive had acquired pointed or serendipitous observations with $\geq 2 \mathrm{ks}$ exposures for $\sim 80$ PNe. These observations were analyzed, and X-ray emission from 13 PNe was detected (Guerrero, Chu, \& Gruendl 2000). Of these $13 \mathrm{PNe}$, only three had been reported to show extended emission that could be associated with hot gas: NGC 6543 (Kreysing et al. 1992), A 30 (Chu \& Ho 1995; Chu, Chang, \& Conway 1997), and BD +30³639 (Leahy, Kwok, \& Yin 2000). In all three cases the X-ray emission was only marginally more extended than the point spread function of the detector used.

An indirect indication of diffuse X-ray emission from hot gas in PNe is provided by the ROSAT PSPC spectra. The PNe detected by ROSAT show three types of spectra (Conway \& Chu 1997; Guerrero, Chu, \& Gruendl 2000). Type 1 spectra, peaking near $0.1-0.2 \mathrm{keV}$ and diminishing above $0.5 \mathrm{keV}$, are consistent with photospheric emission from hot CSPNs. Type 2 spectra, peaking at $\geq 0.5$ $\mathrm{keV}$, are characteristic for plasma emission. Type 3 spectra, possessing a bright peak near $0.1-0.2 \mathrm{keV}$ and a weak peak at $\sim 1 \mathrm{keV}$, are apparently composites of the first two types. Objects with Type 2 spectra are good candidates for diffuse X-ray emission; these include $\mathrm{BD}+30^{\circ} 3639$ and NGC 6543 , and possibly NGC 7009. $A S C A$ observations of $\mathrm{BD}+30^{\circ} 3639$ have provided high-resolution spectra and confirmed the nature of plasma emission (Arnaud, Borkowski, \& Harrington 1996).

Finally, the superb angular resolution of the Chandra X-ray Observatory made it possible to unambiguously resolve the diffuse X-ray emission from $\mathrm{PN}$ interiors: $\mathrm{BD}+30^{\circ} 3639$, NGC 6543, and NGC 7027 (Kastner et al. 2000, 2001; Chu et al. 2001). The unprecedented sensitivity of XMM-Newton made it possible to resolve the faint diffuse X-ray emission from NGC 7009 (Guerrero, Gruendl, \& Chu 2002). These observations allow us to examine the distribution and physical conditions of the hot gas in PN interiors.

Table 2 lists all PNe that have been reported to emit X-rays, including a PN that was detected after the X-ray source (RXJ 2117.1+3412) and a PN in the SMC. The possible detections and the mis-identified sources are listed at the bottom of the table. The majority of the valid X-ray sources in PNe consist of X-ray emission from their photospheres, showing Type 1 or Type 3 ROSAT PSPC spectra. Objects showing Type 2 ROSAT PSPC spectra are the best candidates for diffuse X-ray emission. References are given for each object. 
Table 2. PNe with Reported X-ray Emission

\begin{tabular}{|c|c|c|c|c|}
\hline PN Name & Observations & $\begin{array}{l}\text { ROSAT } \\
\text { PSPC } \\
\text { Spectrum }\end{array}$ & $\begin{array}{l}\text { Diffuse } \\
\text { Emission }\end{array}$ & Reference \\
\hline \multicolumn{5}{|c|}{ PNe with X-ray Sources } \\
\hline A 30 & $R O S A T$ & Type 1 & Yes? & 12,17 \\
\hline A 36 & $E X O S A T, R O S A T$ & Type $2 ?$ & & 3,19 \\
\hline $\mathrm{BD}+30^{\circ} 3639$ & $\begin{array}{l}\text { ROSAT,ASCA } \\
\text { Chandra }\end{array}$ & Type 2 & Yes & $\begin{array}{l}6,14,20 \\
21\end{array}$ \\
\hline K $1-16$ & $R O S A T$ & Type 1 & & 13,19 \\
\hline K $1-27$ & $R O S A T$ & Type $2 / 3 ?$ & & 11 \\
\hline LoTr 5 & $E X O S A T, R O S A T$ & Type 3 & & $5,6,13,19$ \\
\hline NGC 246 & $\begin{array}{l}\text { Einstein,EXOSAT, } \\
\text { ROSAT }\end{array}$ & Type 1 & & $\begin{array}{l}2,3 \\
13\end{array}$ \\
\hline NGC 1360 & $\begin{array}{l}\text { Einstein,EXOSAT, } \\
R O S A T\end{array}$ & Type 1 & & $\begin{array}{l}1,2,3 \\
13\end{array}$ \\
\hline NGC 4361 & $E X O S A T, R O S A T$ & & & 3,6 \\
\hline NGC 3587 & EXOSAT,ROSAT & Type 1 & & $3,15,18$ \\
\hline NGC 6543 & ROSAT,Chandra & Type 2 & Yes & 6,22 \\
\hline NGC 6853 & $\begin{array}{l}\text { Einstein,EXOSAT, } \\
R O S A T\end{array}$ & Type 1 & & $\begin{array}{l}2,3 \\
6,8,13\end{array}$ \\
\hline NGC 7027 & Chandra & & Yes & 25 \\
\hline NGC 7009 & $R O S A T, X M M$ & Type 2 & Yes & 19,26 \\
\hline NGC 7293 & $\begin{array}{l}\text { Einstein,EXOSAT, } \\
R O S A T, C h a n d r a\end{array}$ & Type 3 & & $\begin{array}{l}2,3 \\
10,15,23,24\end{array}$ \\
\hline RXJ 2117.1+3412 & $R O S A T$ & Type 1 & & 7,9 \\
\hline $\mathrm{N} 67$ in the SMC & Einstein & & & 4 \\
\hline
\end{tabular}

PNe with Possible (2 $\sigma)$ X-ray Sources

NGC 2371-72

$R O S A T$

19

NGC 2392

$R O S A T$

19

NGC 6572

ROSAT

19

PNe with Mis-identified X-ray Sources
A 12
ROSAT
6,13
A 33
Einstein, ROSAT
$2,16,19$
NGC 1535
EXOSAT,ROSAT
$3,15,18$ 
References.- (1) de Korte et al. 1985; (2) Tarafdar \& Apparao 1988; (3) Apparao \& Tarafdar 1989; (4) Wang 1991; (5) Apparao, Berthiaume, \& Nousek 1992 (6) Kreysing et al. 1992; (7) Appleton, Kawaler, \& Eitter 1993. (8) Chu, Kwitter, \& Kaler 1993; (9) Motch, Werner, \& Pakull 1993. (10) Leahy, Zhang, \& Kwok 1994; (11) Rauch, Köppen, \& Werner 1994; (12) Chu \& Ho 1995; (13) Hoare et al. 1995; (14) Arnaud, Borkowski, \& Harrington 1996; (15) Leahy et al. 1996; (16) Conway \& Chu 1997; (17) Chu, Chang, \& Conway 1997; (18) Chu, Gruendl, \& Conway 1998; (19) Guerrero, Chu, \& Gruendl 2000; (20) Leahy, Kwok, \& Yin 2000; (21) Kastner et al. 2000; (22) Chu et al. 2001; (23) Gruendl et al. 2001; (24) Guerrero et al. 2001; (25) Kastner, Vrtilek, \& Soker 2001; (26) Guerrero, Gruendl, \& Chu 2002.

\subsection{Physical Properties of Hot Gas in PNe}

Chandra and XMM-Newton observations have resolved diffuse X-ray emission from four PNe. The X-ray contour maps and spectra of BD $+30^{\circ} 3639$, NGC 6543, and NGC 7027 are presented in Figure 1, and those of NGC 7009 in Figure 1 of Guerrero, Gruendl, \& Chu (2002, this volume). The physical parameters of these four PNe are summarized in Table 3, where the distance $(d)$ and size of the nebula, temperature $\left(T_{\mathrm{e}}\right)$ and rms density $\left.\left(<N_{\mathrm{e}}\right\rangle\right)$ of the hot gas, absorption column density $\left(N_{\mathrm{H}}\right)$, and X-ray luminosity $\left(L_{\mathrm{X}}\right)$ are given. Despite the uncertainty in distances and the large range of absorption column density, it appears that the temperature, density, and X-ray luminosity decrease with increasing nebular size. This trend suggests an evolutionary effect: PNe are brightest in $\mathrm{X}$-rays when they are young.

Table 3. Properties of PNe with Extended X-ray Emission

\begin{tabular}{lcccccc}
\hline \hline PN Name & $\begin{array}{c}d \\
{[\mathrm{kpc}]}\end{array}$ & $\begin{array}{c}\text { size } \\
{[\mathrm{pc}]}\end{array}$ & $\begin{array}{c}T_{\mathrm{e}} \\
{\left[10^{6} \mathrm{~K}\right]}\end{array}$ & $\begin{array}{c}<N_{\mathrm{e}}> \\
{\left[\mathrm{cm}^{-3}\right]}\end{array}$ & $\begin{array}{c}N_{\mathrm{H}} \\
{\left[\mathrm{cm}^{-2}\right]}\end{array}$ & $\begin{array}{c}L_{\mathrm{X}} \\
{\left[\mathrm{ergs} \mathrm{s}^{-1}\right]}\end{array}$ \\
\hline BD +30 3639 & 1 & $0.025 \times 0.02$ & 2.7 & 200 & $1 \times 10^{21}$ & $1.6 \times 10^{32}$ \\
NGC 7027 & 0.9 & $0.04 \times 0.03$ & 3.0 & 150 & $6 \times 10^{21}$ & $1.3 \times 10^{32}$ \\
NGC 6543 & 1 & $0.05 \times 0.04$ & 1.7 & $\sim 50$ & $9 \times 10^{20}$ & $1.0 \times 10^{32}$ \\
NGC 7009 & 1.2 & $0.145 \times 0.06$ & 1.8 & $\sim 25$ & $8 \times 10^{20}$ & $3 \times 10^{31}$ \\
\hline \hline
\end{tabular}

\section{UV Views of Hot Gas in Planetary Nebulae}

If $\mathrm{C}^{+3}, \mathrm{~N}^{+4}$, and $\mathrm{O}^{+5}$ are produced by collisional ionization, the $\mathrm{C}$ IV $\lambda \lambda 1548$, $1550, \mathrm{~N}$ V $\lambda \lambda 1238,1242$, and O VI $\lambda \lambda 1031,1037$ lines can be used to diagnose the presence of $1 \times 10^{5} \mathrm{~K}, 2 \times 10^{5} \mathrm{~K}$, and $3 \times 10^{5} \mathrm{~K}$ gas, respectively. Note, however, that $\mathrm{C}^{+2}, \mathrm{~N}^{+3}$, and $\mathrm{O}^{+4}$, having ionization potentials of $47.9 \mathrm{eV}, 77.5 \mathrm{eV}$, and $113.9 \mathrm{eV}$, can be photoionized by stars with effective temperatures higher than $35,000 \mathrm{~K}, 75,000 \mathrm{~K}$, and $125,000 \mathrm{~K}$, respectively. Therefore, UV absorption line studies of $10^{5} \mathrm{~K}$ gas need to be carefully designed to minimize the confusion caused by photoionization.

High-dispersion UV observations of nebular $\mathrm{C}$ IV and $\mathrm{N} V$ absorption lines in PNe have been made with the International Ultraviolet Explorer (IUE) and Hubble Space Telescope (HST). For example, IUE observations of NGC6543 (Pwa, Mo, \& Pottasch 1984) and HST GHRS observations of A 78 (Harrington, 

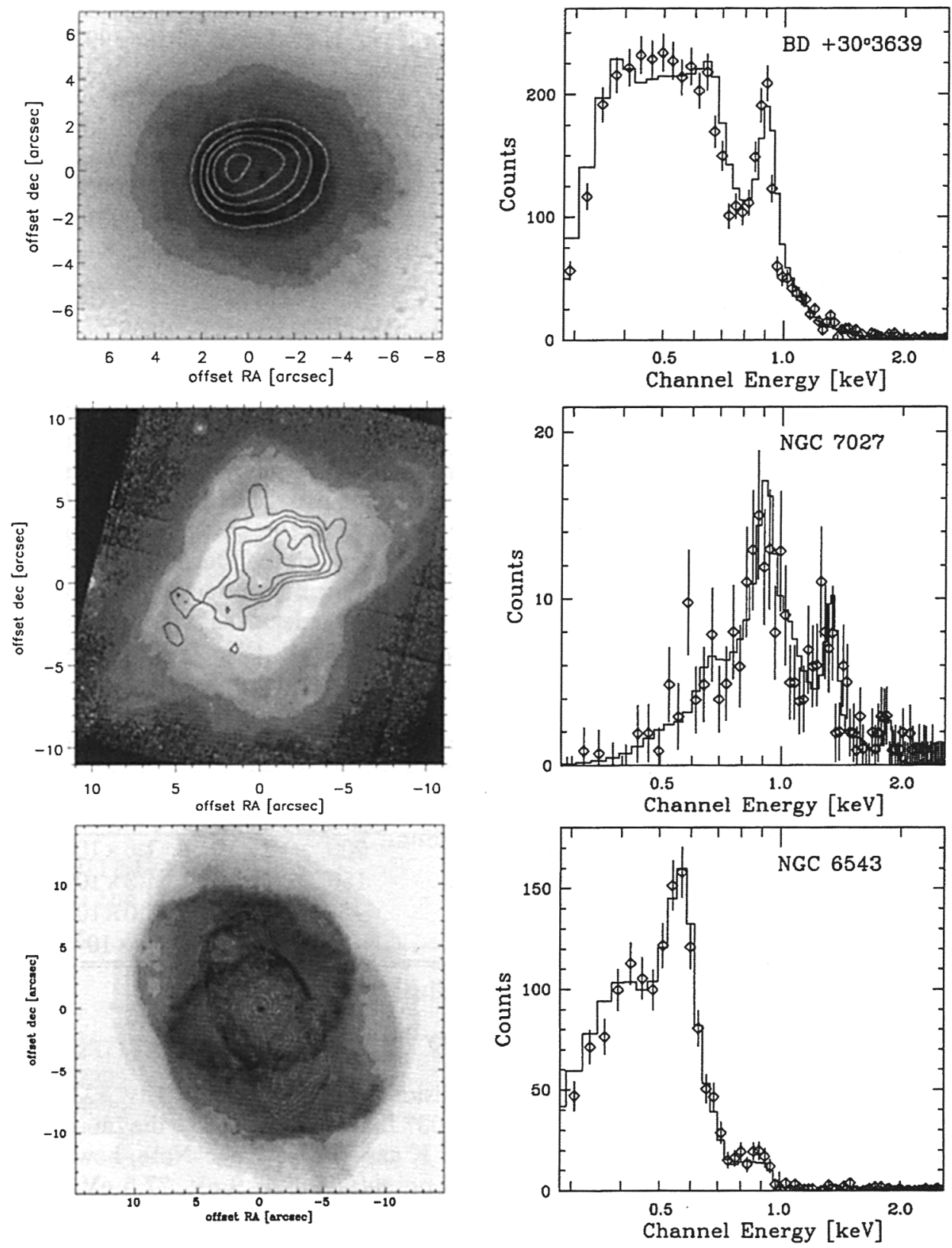

Figure 1. Chandra ACIS contour maps and spectra of three PNe, taken from Kastner et al. (2000, 2001) and Chu et al. (2001). The X-ray contours are overlaid on $\mathrm{H} \alpha$ images for $\mathrm{BD}+30^{\circ} 3639$ and NGC 6543, and an $\mathrm{H}_{2} 2.12 \mu \mathrm{m}$ image for NGC 7027. 
Borkowski, \& Tsvetanov 1995) showed nebular C IV absorption. As the He II or $\mathrm{H}$ Zanstra temperatures of their central stars are $\sim 50,000 \mathrm{~K}$ and $\sim 70,000$ K, respectively (Kaler \& Jacoby 1991; Kaler 1983), these observations are not useful for the study of $10^{5} \mathrm{~K}$ gas. $\mathrm{N} \mathrm{V}$ lines have small oscillator strengths, so are difficult to detect.

$\mathrm{O}$ VI lines in the far-UV, being strong and less affected by photoionization, are the best lines to study $10^{5} \mathrm{~K}$ gas. High-dispersion spectroscopic observations of the $\mathrm{O}$ vi lines have been made with the Orbiting Retrievable Far and Extreme UV Spectrometer (ORFEUS) for only the brightest CSPNs, e.g., NGC 6543 (Zweigle et al. 1997). The spectral resolution of $O R F E U S, \lambda / \Delta \lambda=$ 3000 , was not adequate to distinguish clearly among the interstellar and nebular $\mathrm{O}$ VI and $\mathrm{H}_{2}$ absorption lines. It was not clear whether nebular $\mathrm{O}$ vI absorption was present in the spectrum of NGC 6543.

Finally, the Far-UV Spectroscopic Explorer (FUSE) (Sonneborn 2002) provides the sensitivity and resolution required to study the nebular O VI absorption and emission lines for a large number of PNe. We have initiated FUSE programs to study the hot gas at the interfaces in PNe. We have obtained FUSE observations for seven CSPNs for a wide range of stellar temperatures. The spectral region around the $\mathrm{O}$ VI lines of these observations is presented in Figure 2. This figure demonstrates clearly that the stellar and interstellar/nebular lines are very complex. The spectra of NGC 2392 and NGC 6058 show narrow absorption components at expected locations for both $\mathrm{O}$ VI $\lambda \lambda 1031,1037$ lines, and thus provide the most promising candidates for detections of the $10^{5} \mathrm{~K}$ gas at the interface. We are in the process of enlarging our sample to include all archival FUSE observations of CSPNs in order to empirically sort out the temperature dependence of the spectral features of the stars and to pinpoint the nebular absorption components (Gruendl et al., in preparation).

\section{Concluding Remarks}

Chandra and XMM-Newton observations have unambiguously detected diffuse $\mathrm{X}$-ray emission from the hot gas in PN interiors, illustrating that PNe are excellent laboratories to study shocked stellar winds and the interfaces between this hot gas and cool nebular shells. Preliminary results show that the hot gas is overpressurized and drives the nebular expansion. It is puzzling, however, that the shocked stellar winds in PNe can cool from $10^{7}-10^{8} \mathrm{~K}$ to $2-3 \times 10^{6} \mathrm{~K}$ within the short dynamic age of the nebular shells, 1,000 yrs. Future Chandra and $X M M-N e w t o n$ observations of hot interior gas, in conjunction with FUSE observations of the interfaces, for $\mathrm{PNe}$ with a wide range of morphologies and evolutionary stages are needed to empirically establish our knowledge of the physical conditions and evolution of the hot gas in PN interiors. A sound knowledge of the hot gas in PN interiors is crucial to our understanding of the formation and evolution of $\mathrm{PNe}$ as a whole.

Acknowledgments. We gratefully acknowledge the observing times we received from Chandra, XMM-Newton, and FUSE. This work has been supported by NASA grants associated with these observing programs: SAO GO 0-1004X, NAG 5-10042, and NAG 5-10182. 


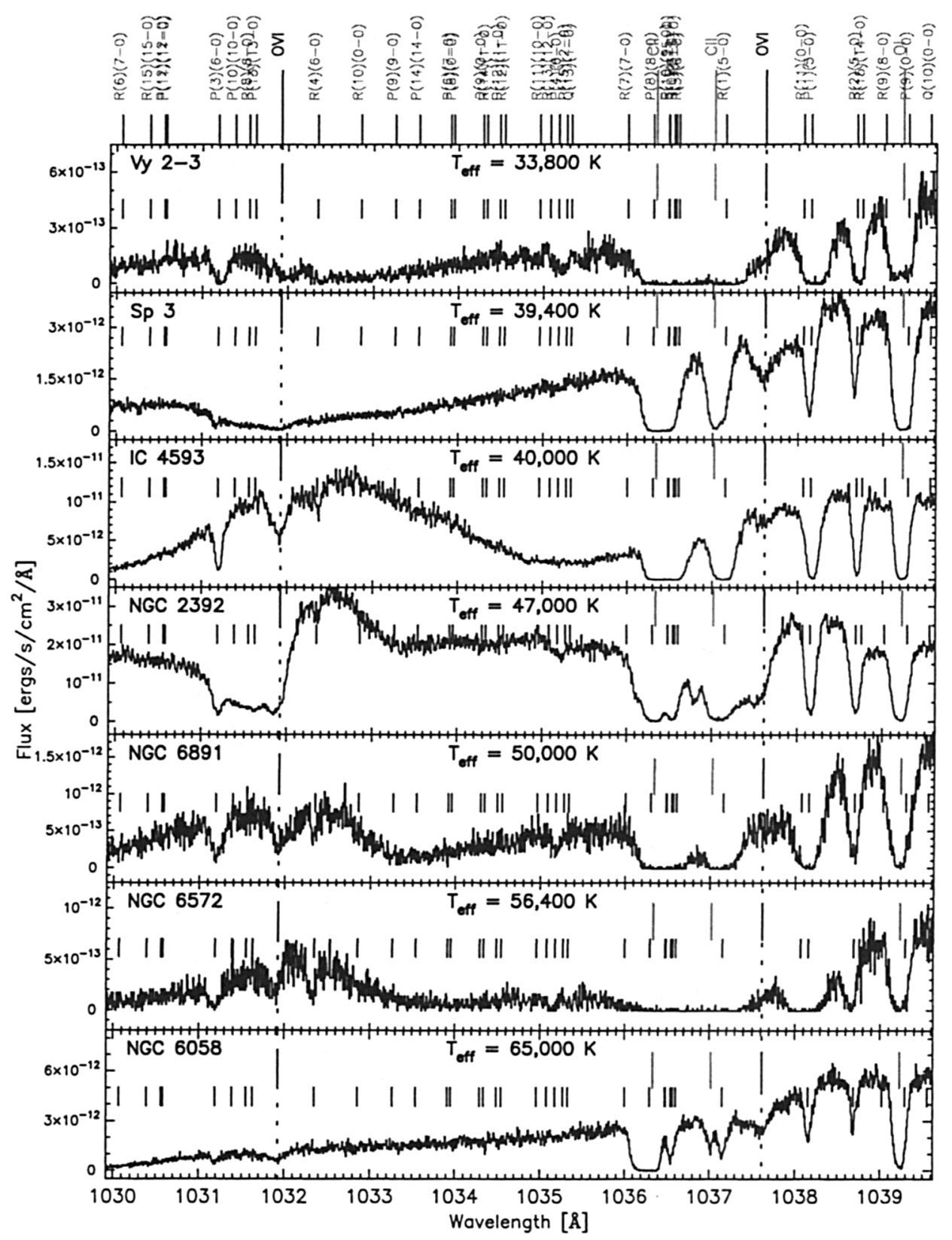

Figure 2. FUSE spectra of seven CSPNs in the O VI lines. The spectra are arranged according to the stellar $T_{\text {eff. }}$ The location of O VI, $\mathrm{C}$ II, $\mathrm{O} \mathrm{I}$, and $\mathrm{H}_{2}$ lines are marked on the top margin. 


\section{References}

Apparao, K. M. V., \& Tarafdar, S. P. 1989, ApJ, 344, 826

Apparao, K. M. V., Berthiaume, G. D., \& Nousek, J. A. 1992, ApJ, 397, 534

Appleton, P. N., Kawaler, S. D., \& Eitter, J. J. 1993, AJ, 106, 1973

Arnaud, K., Borkowski, K. J., \& Harrington, J. P. 1996, ApJ, 462, L75

Chu, Y.-H., Chang, T. H., \& Conway, G. M. 1997, ApJ, 482, 891

Chu, Y.-H., Gruendl, R. A., \& Conway, G. M. 1998, AJ, 116, 1882

Chu, Y.-H., Guerrero, M. A., Gruendl, R. A., Williams, R. M., \& Kaler, J. B. 2001, ApJ, 553, L69

Chu, Y.-H., \& Ho, C.-H. 1995, ApJ, 448, L127

Chu, Y.-H., Kwitter, K., \& Kaler, J. 1993, AJ, 106, 650

Conway, G. M., \& Chu, Y.-H. 1997, in IAU Symp. 180, Planetary Nebulae, ed. H. J. Habing, \& H. J. G. L. M. Lamers (Dordrecht: Kluwer), 214

de Korte, P. A. J., Claas, J. J., Jansen, F. A., \& McKechnie, S. P. 1985, Adv. Space Res., 5,57

Gruendl, R. A., Chu, Y.-H., O’Dwyer, I. J., \& Guerrero, M. 2001, AJ, 122, 308

Guerrero, M. A., Chu, Y.-H., \& Gruendl, R. A. 2000, ApJS, 129, 295

Guerrero, M. A., Chu, Y.-H., Gruendl, R. A., Williams, R. M., \& Kaler, J. B. 2001, ApJ, 553, L55

Guerrero, M. A., Gruendl, R. A., \& Chu, Y.-H. 2002, A\&A, in press; also in this volume Harrington, J. P., Borkowski, K. J., \& Tsvetanov, Z. 1995, ApJ, 439, 264

Hoare, M.G., Martin, A.B., Werner, K., \& Fleming, T. 1995, MNRAS, 273, 812

Kaler, J. B. 1983, ApJ, 271, 188

Kaler, J. B., \& Jacoby, G. H. 1991, ApJ, 372, 215

Kastner, J. H., Soker, N., Vrtilek, S. D., \& Dgani, R. 2000, ApJ, 545, L57

Kastner, J. H., Vrtilek, S. D., \& Soker, N. 2001, ApJ, 550, L189

Kreysing, H. C., Diesch, C., Zweigle, J., Staubert, R., Grewing, M., \& Hasinger, G. 1992, A\&A, 264, 623

Kwok, S., Purton, C. R., \& FitzGerald, P. M. 1978, ApJ, 219, L125

Leahy, D. A., Kwok, S., \& Yin, D. 2000, ApJ, 540, 442

Leahy, D. A., Zhang, C. Y., \& Kwok, S. 1994, ApJ, 422, L205

Leahy, D. A., Zhang, C. Y., Volk, K., \& Kwok, S. 1996, ApJ, 466, L352

Motch, C., Werner, K., \& Pakull, M. W. 1993, A\&A, 268, 561

Pwa, T. H., Mo, J. E., \& Pottasch, S. R. 1984, A\&A, 139, L1

Rauch, T., Köppen, J., \& Werner, K. 1994, A\&A, 286, 543

Sonneborn, G. 2002, in this volume

Tarafdar, S. P., \& Apparao, K. M. V. 1988, ApJ, 327, 34

Wang, Q. 1991, MNRAS, 252, 47p

Weaver, R., McCray, R., Castor, J., Shapiro, P., Moore, R. 1977, ApJ, 218, 377

Zweigle, J. et al. 1997, A\&A, 321, 891 


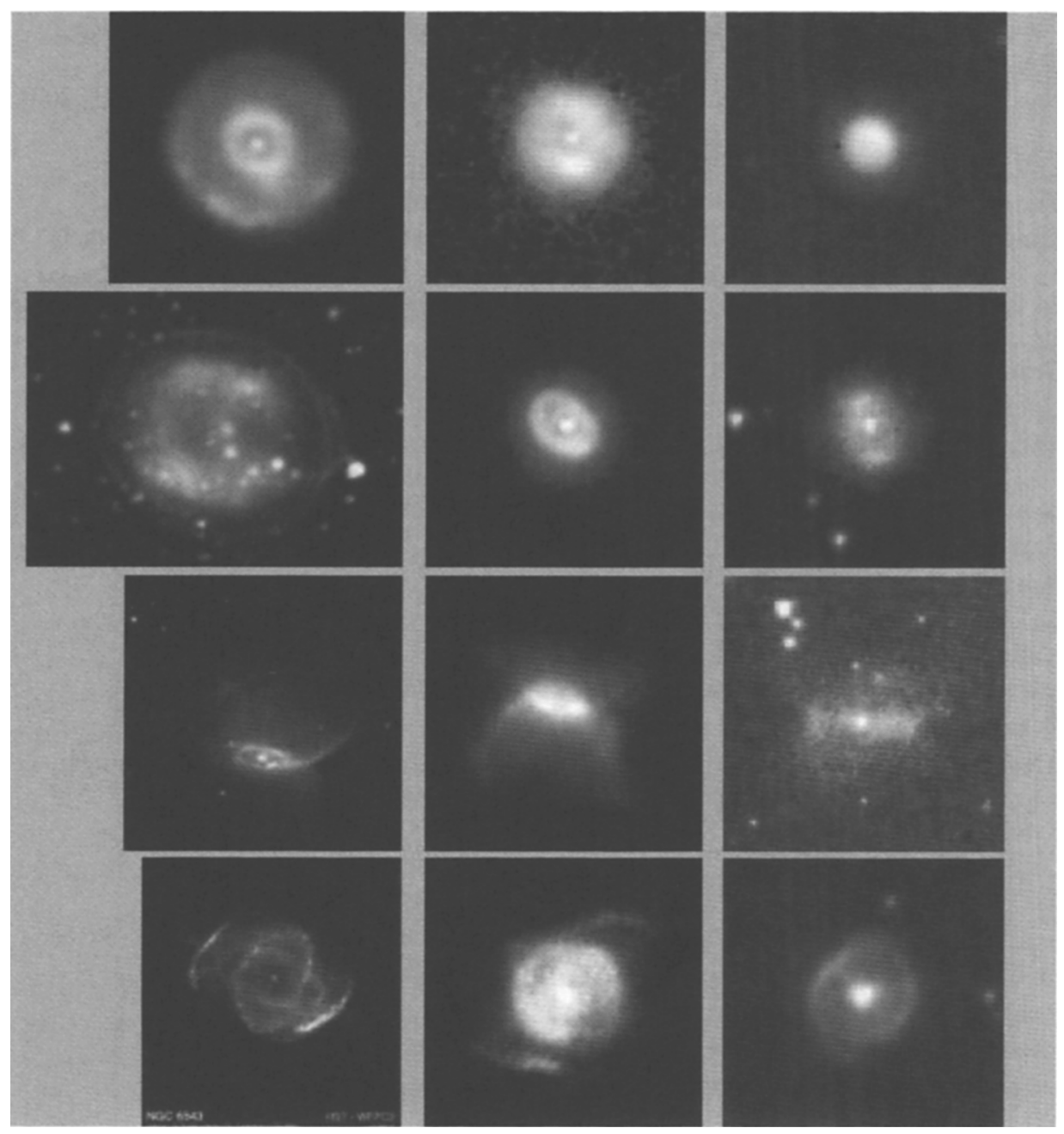

PNe have a variety of morphologies, as illustrated in these HST images of 12 galactic PNe. Images courtesy of L Stanghellini. 